\title{
A comparison of the effects of spatial separation on apparent motion in the auditory and visual modalities
}

\author{
THOMAS Z. STRYBEL \\ California State University, Long Beach, California \\ and \\ CAROL L. MANLIGAS, OZZIE CHAN, and DAVID R. PERROTT \\ California State University, Los Angeles, California
}

\begin{abstract}
In the present investigation, the effects of spatial separation on the interstimulus onset intervals (ISOIs) that produce auditory and visual apparent motion were compared. In Experiment 1, subjects were tested on auditory apparent motion. They listened to 50-msec broadband noise pulses that were presented through two speakers separated by one of six different values between $0^{\circ}$ and $160^{\circ}$. On each trial, the sounds were temporally separated by 1 of 12 ISOIs from 0 to 500 msec. The subjects were instructed to categorize their perception of the sounds as "single," "simultaneous," "continuous motion," "broken motion," or "succession." They also indicated the proper temporal sequence of each sound pair. In Experiments 2 and 3, subjects were tested on visual apparent motion. Experiment 2 included a range of spatial separations from $6^{\circ}$ to $80^{\circ}$; Experiment 3 included separations from $.5^{\circ}$ to $10^{\circ}$. The same ISOIs were used as in Experiment 1 . When the separations were equal, the ISOIs at which auditory apparent motion was perceived were smaller than the values that produced the same experience in vision. Spatial separation affected only visual apparent motion. For separations less than $2^{\circ}$, the ISOIs that produced visual continuous motion were nearly equal to those which produced auditory continuous motion. For larger separations, the ISOIs that produced visual apparent motion increased.
\end{abstract}

Apparent motion is an illusion produced by the proper timing and placement of two discrete stimuli; under optimal conditions, movement of the lead stimulus toward the lag stimulus is perceived. Apparent motion was first demonstrated by Exner in 1875, but it was Wertheimer's (1912) article that initiated much interest in the phenomenon. In 1917, Burtt demonstrated that the illusion of apparent motion could also occur in the auditory and tactual modalities (Burtt, 1917a, 1917b). Most subsequent investigations of apparent motion, however, have been focused on the visual illusion. No direct comparisons between the modalities of apparent motion have been reported. Our purpose in the present investigation was to compare the visual modality of the illusion of apparent motion with the auditory modality. At the same time, we examined the effects of the spatial separation between the stimuli that were used in each modality.

\section{Visual Apparent Motion}

Soon after Wertheimer's seminal paper, Korte published what have been called the "laws" of apparent motion, which describe the relationships between the "primary"

This research was supported in part by grants from the National Science Foundation (BNS-8512317) and the National Institute of Health (3SO6 RR0801-1452). Carol L. Manligas is now in the Department of Psychology at the University of Georgia, Athens, GA 30602 . Correspondence should be addressed to Thomas Z. Strybel, Department of Psychology, California State University, Long Beach, CA 90840. variables that affect the visual illusion (Korte, 1915). These variables include the exposure time of each stimulus, as well as the temporal and spatial separation between the stimuli. Korte's third law states that as this spatial separation increases, the interstimulus interval (ISI) must also increase if apparent motion is to be maintained (with the exposure time held constant). This "law" has been tested several times, and some modifications to it have been proposed (see Kolers, 1972). For example, Neuhaus (1930) reported that apparent motion can be seen for a range of ISIs, and that as the spatial separation increases, the range of the ISIs that produce apparent motion decreases.

More recent work (e.g., Anstis, 1978; Braddick, 1974) has suggested that the spatial separation of the stimuli affects apparent motion only when the separation is greater than $20^{\circ}$ of visual angle when the stimuli are presented to the foveal region of the eye. This value of spatial separation increases with increasing eccentricity. The differential effect of spatial separation has led to the development of a two-process theory of motion perception. Baker and Braddick (1985) suggest that the effect of spatial separation on the ISI range that produces apparent motion, as proposed by Korte (1915) and Neuhaus (1930), describes the long-range motion detecting mechanism only.

\section{Auditory Apparent Motion}

As a result of Burtt's (1917a) demonstration that apparent motion could be perceived with two auditory 
stimuli, it has generally been assumed that some aspects of a moving stimulus are processed similarly in each modality. However, the similarities and differences between these illusions have not really been elaborated. Briggs and Perrott (1972) reported that auditory apparent motion is heard at ISIs lower than those at which the visual illusion is perceived, and that the relationship between exposure time and ISI in audition is similar to that described by Korte (1915) for the visual modality. Strybel, Manligas, and Perrott (1989) found no effects of spatial separation on the ISI values that produce auditory apparent motion under binaural listening conditions, for separations between $10^{\circ}$ and $160^{\circ}$. Yet although such similarities and differences between the visual and auditory illusion have been noticed, it is difficult to determine how much the differences have simply resulted from different experimental conditions. For example, how can one compare the factors influencing the apparent motion of an auditory stimulus that is heard to sweep through the head of the listener (under dichotic listening conditions) or to sweep across a darkened room (under free-field listening conditions) with the factors involved in the apparent visual motion of various figures presented on a CRT or a tachistoscope? In addition, various response measures have been used, within modalities as well as between them (e.g., scales of motion, percentage of motion reported, and direction of motion), and the relationships between these response measures have not been documented.

In the present investigation, we therefore explored the relationship between ISI and spatial separation in both the auditory and the visual modalities under nearly identical conditions. We conducted three experiments. In Experiment 1 , auditory apparent motion was measured under a large range of interstimulus onset intervals (ISOIs; 0$500 \mathrm{msec}$ ) and spatial separations $\left(6^{\circ}-160^{\circ}\right) .{ }^{1}$ In Experiment 2, visual apparent motion was measured under the same range of ISOIs and a range of spatial separations between $6^{\circ}$ and $80^{\circ}$. In Experiment 3, visual apparent motion was measured for a range of separations more typical of visual apparent motion experiments $\left(.5^{\circ}-8^{\circ}\right)$.

\section{METHOD}

\section{Subjects}

Eight subjects were tested in Experiment 1 ( 6 men and 2 women). None of them had any prior experience of either apparent motion or auditory localization tasks, and all reported normal hearing. In Experiment 2, to examine visual apparent motion, we tested 8 subjects -4 from the auditory experiment, and 4 who were experimentally naive. All had normal or corrected-to-normal vision. Five subjects were tested in Experiment 3, 3 of whom were naive and 2 of whom were experienced from Experiment 2.

\section{Apparatus}

Testing was conducted in an audiometric chamber (IAC Model 1200 ), with the walls modified to produce a semianechoic environment. The subject was seated in a chair that had been modified to allow the placement of speakers (or lights) at any desired azimuth angle and any distance from the subject up to $3 \mathrm{~m}$. The speakers were hung at the subject's ear level.
In Experiment 1, broadband noise (500-8000 Hz) was produced by two Grason-Stadler Model 901B noise generators. Each signal was led through a separate channel of an Outland Co. band-pass filter, through an electronic switch, to a separate Realistic $62-\mathrm{cm}$ speaker located in the test chamber. The duration of each stimulus was $50 \mathrm{msec}$, and the sound level was $60 \mathrm{~dB}$ A-weighted. All timing was controlled by programmable modules (Coulbourn Instruments) and a microprocessor (Commodore 64) located in a room adjacent to the sound chamber. The microprocessor also controlled response collection by means of a response box attached to the game port of the computer. The response box contained five three-position spring-loaded switches.

For the visual apparent motion trial blocks in Experiment 2, the following modifications were made to the apparatus: The speakers were replaced with small light bulbs, which were enclosed in a red lens ( $1.2 \mathrm{~cm}$ in diameter) and mounted in the positions previously occupied by the speakers. The illumination of the lights was $1 \mathrm{fc}$, measured $7.6 \mathrm{~cm}$ from the source. ${ }^{2}$ In this experiment, a small background light was added to ensure that the adaptation level of the subject remained constant during each trial block. The ambient illumination was approximately $.06 \mathrm{fc}$. A small white cross, which served as a fixation point, was placed directly in front of the subject slightly beyond the distance of the lights. The electronic switches were replaced with a Coulbourn Instruments lamp driver.

To achieve the small separations required in Experiment 3, the light bulb and lens arrangement was replaced by small diodes. The illumination of these diodes was $.5 \mathrm{fc}$, measured $.64 \mathrm{~cm}$ from the source.

\section{Procedure}

Six different spatial separations were used in Experiment $1\left(6^{\circ}\right.$, $10^{\circ}, 20^{\circ}, 40^{\circ}, 80^{\circ}$, and $160^{\circ}$ ), centered at the subject's midline, at a distance of $.86 \mathrm{~m}$ from the subject. All trials were run at a single speaker location, and the order of speaker positions was randomized for each subject. At each spatial separation, there were 12 ISOIs $(0,10,20,30,45,75,90,105,130,200,300$, and $500 \mathrm{msec}$ ). In Experiment 2, five values of spatial separation were tested $\left(6^{\circ}, 10^{\circ}, 20^{\circ}, 40^{\circ}\right.$, and $\left.80^{\circ}\right)$, and the same ISOI values were used at each separation. In Experiment 3, five values of separation were tested $\left(.5^{\circ}, 1^{\circ}, 2^{\circ}, 4^{\circ}\right.$, and $\left.8^{\circ}\right)$. For all three experiments, each ISOI was presented 30 times at each separation, 15 originating on the subject's right, and 15 on the left. Therefore, a trial block consisted of 360 trials presented to the subject at a particular speaker position in a random sequence.

The subjects were instructed to listen to each stimulus presentation and classify their perception of the stimulus pair into one of five categories: Single-a single sound or light was heard or seen somewhere, with no movement perceived; simultaneous-two sounds or lights were heard or seen at the same time, with no movement between them; continuous motion-the sound or light started at one side and moved continuously to the other side; broken motion-the sound or light started at one side and moved toward the other side, but the movement was discontinuous, broken or interrupted; and succession-two sounds or lights were perceived successively, with no movement between them. These categories, developed by Briggs and Perrott (1972) for the study of auditory apparent motion, have been used in subsequent auditory apparent motion experiments (e.g., Perrott, 1974; Strybel et al., 1989).

In addition to classifying their perceptions of the stimuli, the subjects also indicated whether the first sound or light originated to the left or the right. Their judgment involved determining the apparent direction of the motion on trials that produced apparent motion, or the proper temporal sequence on trials that did not. Each category selection was indicated with an appropriately labeled switch, which the subjects threw either to the left or the right in order to indicate the direction of origination. When they did not perceive any direction, the subjects were to guess. 
Prior to a trial block, each subject read the instructions and was allowed to question the experimenter about the categories of response. At the beginning of each session, the subject sat down in the chair and then reviewed the instructions provided. In the auditory experiment, a black cloth was hung in front of the subject's face so that the positions of the speakers could not be seen before testing began. Testing in the auditory experiment was conducted in the dark; in the visual experiment, on the other hand, a low level of illumination was provided.

The illumination of a small light mounted on the response box indicated that the subject should initiate the test session by throwing any switch on the response box. The light was then turned off, and $500 \mathrm{msec}$ later the first stimulus was presented. Five hundred milliseconds after the presentation, the light was again illuminated, signaling to the subject to make a response. After the response was made, the light was turned off, and $500 \mathrm{msec}$ later the next stimulus was presented. The task was thus self-paced: The subject could delay the presentation of a stimulus by delaying the response.

\section{RESULTS}

In Experiment 2, which involved visual apparent motion at large separations, data from 1 naive and $1 \mathrm{ex}-$ perienced subject were not included in further analysis. The naive subject failed to report motion at any separation tested. The experienced subject, who was inadvertently provided with an alternate set of instructions on several trial blocks, reported little motion. Therefore, the analysis of auditory apparent motion (Experiment 1) was based on 8 subjects, but the analysis of visual apparent motion at large separations (Experiment 2) was based on 6 subjects.

The percentage of trials on which a category was assigned to each ISOI was determined for each subject at every separation at each modality. In all conditions, very few "single" responses were made. The condition that produced the most "single" responses was the $6^{\circ}$ separation in the auditory experiment. When the ISOI was $0 \mathrm{msec}, 53 \%$ of the responses on the average were "single," and this value decreased to $16 \%$ at the next highest ISOI (10 msec). In the visual experiments, no "single" responses were made at any angle-ISOI combination. For all modalities and separations, at small ISOIs the dominant response was "simultaneous." As the ISOI increased, the response changed to "continuous motion," then "broken motion," and finally "succession" at the largest ISOIs. It should be noted that at some ISOIs, however, there was some overlap between categories.

Figure 1 presents the mean percentage of continuous motion reports for Experiments 1 and 2 . From this figure, it is obvious that auditory apparent motion occurs at smaller ISOIs than visual motion does. In addition, the peaks of the auditory distributions are considerably higher than the visual curves, and the range of ISOIs that produce apparent motion is more restricted in audition.

Figure 2 presents the percentage of broken motion responses for angles, ISOIs, and modalities tested in Experiments 1 and 2 . The differences between modalities

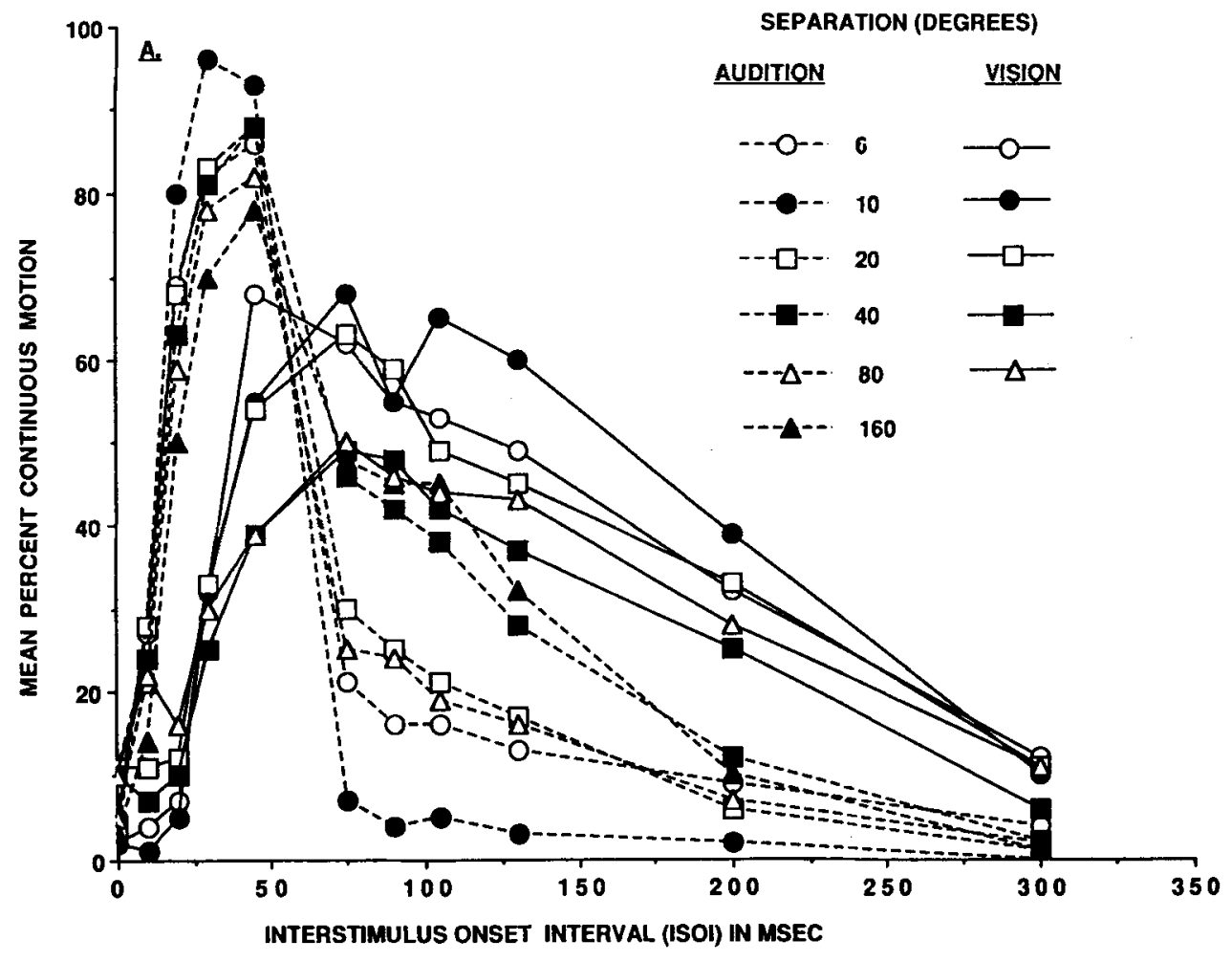

Figure 1. Mean percentage of continuous motion reports for Experiments 1 and 2 (auditory and visual apparent motion) for ISOIs between 0 and $300 \mathrm{msec}$ and spatial separations between $6^{\circ}$ and $160^{\circ}$. 


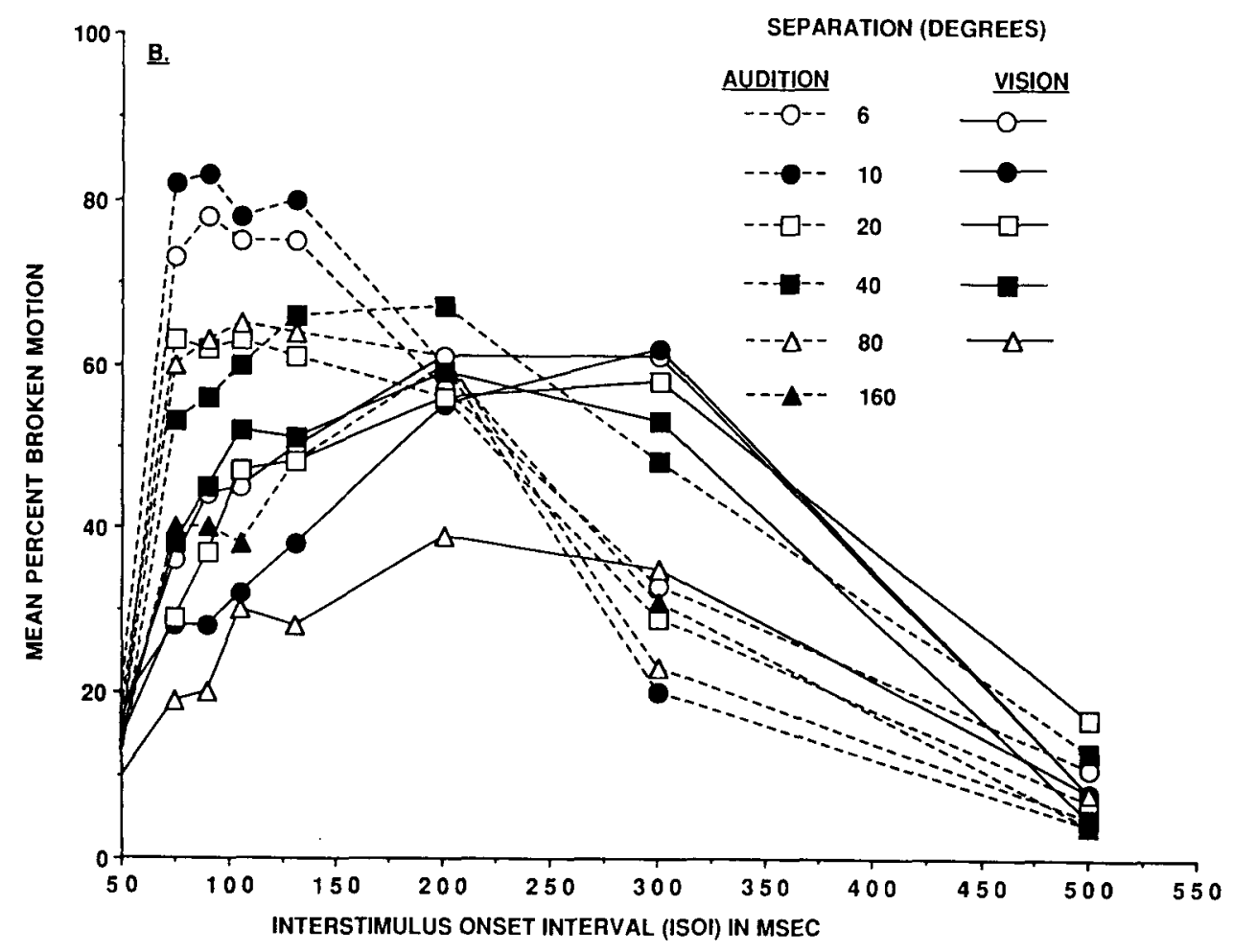

Figure 2. Mean percentage of broken motion reports for Experiments 1 and 2 (auditory and visual apparent motion) for ISOIs between 75 and $500 \mathrm{msec}$ and spatial separations between $6^{\circ}$ and $160^{\circ}$.

are similar to those found with continuous motion. Auditory broken motion is perceived at smaller ISOIs than vision is, and the range of the ISOIs that produce auditory broken motion is more restricted.

An additional difference between auditory and visual apparent motion, evident both in Figures 1 and 2, is that the auditory curves are more leptokurtotic. This is particularly evident in Figure 1. For almost every auditory distribution shown in Figure 1, subjects responded on the average with the continuous motion category at least $80 \%$ of the time at an ISOI of $45 \mathrm{msec}$, but at the next ISOI ( $75 \mathrm{msec}$ ), the percentage of motion responses drops to nearly 0 at small separations and to less than $50 \%$ at the larger separations. In contrast, the visual motion distributions are not as well defined. The peaks of the curves are lower, and the peaks extend across several ISOIs (50$130 \mathrm{msec}$ ). The drop in motion responses at larger ISOIs is more gradual. This could indicate that discrimination between visual continuous and broken motion is more difficult than discrimination between auditory continuous and broken motion.

In both the auditory and visual modalities, however, as the spatial separation increases, the distribution becomes flatter. In the auditory modality, at an ISOI of $45 \mathrm{msec}, 85 \%$ of the responses were "motion" at a separation of $6^{\circ}$. At $160^{\circ}$, this percentage dropped to $78 \%$. In vision, 70\% "continuous motion" responses were found at an ISOI of $45 \mathrm{msec}$ and a separation of $6^{\circ}$. This value dropped to $38 \%$ at a separation of $80^{\circ}$. At an ISOI of $75 \mathrm{msec}$, however, motion was reported $50 \%$ of the time. Thus, in vision, the peak of the function has shifted slightly, and the curve has become flatter. This finding suggests a limit on the separation that produces the impression of motion in each modality. This limit occurs at a smaller separation in vision than it does in audition. In vision, when the separation was $40^{\circ}$ or higher, the percentage of motion responses almost never exceeded $50 \%$.

The effect of separation on broken motion differed slightly in the auditory modality, as is shown in Figure 2. Although the peaks of the curves decrease with increases in spatial separation, for separations between $40^{\circ}$ and $160^{\circ}$, the ISOI has shifted to the range found in vision. At $160^{\circ}$, the auditory broken motion curve is nearly identical to the visual broken motion curves. This is probably the result of an increase in continuous motion responses at longer ISOIs (75-130 msec, shown in Figure 1). In vision, as the separation increases, the peaks of the broken motion curves decrease, until at $80^{\circ}$, broken motion can be seen as having been responded to $39 \%$ of the time, on the average. The range of ISOIs was not affected, however.

These visual distributions in Figure 1 suggest further that when a limit on the separation at which apparent motion can be perceived is approached, the experience of continuous motion will be lost first. Stimuli that are seen as being in continuous motion then become seen as being in broken motion, before the motion per se is lost. For example, in Figure 1 the visual motion distributions at 
$40^{\circ}$ and $80^{\circ}$ are nearly identical. However, the peaks of the broken motion function at $40^{\circ}$ are considerably higher than the $80^{\circ}$ distribution. This suggests that at the $40^{\circ}$ separation, stimuli that were seen as moving at smaller separations are now experienced as being in broken movement. At $80^{\circ}$, however, the peaks of both motion distributions are relatively small; more "succession" and "simultaneous" responses were made.

Figure 3 presents the percentages of continuous $(A)$ and broken (B) motion responses for Experiment 3, in which smaller spatial separations were tested in the visual modality. The auditory distribution at $6^{\circ}$ is included from Experiment 1 for comparison. At the smallest separations, the motion distributions are quite similar to that of the auditory function. For angles between $.5^{\circ}$ and $2^{\circ}$, the peaks of the motion distributions occur at the same ISOI as does the peak of the auditory distribution $(45 \mathrm{msec})$, although the range of ISOIs that produce visual motion is still broader, extending out to roughly $130 \mathrm{msec}$. The motion distributions at $4^{\circ}$ and $8^{\circ}$ are similar to those found at the larger angles in Figure 1. The peaks of the broken motion distributions in Figure 3B are higher at angles of $2^{\circ}$ and less, although the corresponding shift in ISOIs toward the auditory function is not present.

It could be argued, however, that the differences between auditory and visual apparent motion reported here might be the result of differences between subjects. In order to determine how much the obtained results were due to differences in subjects, the data were summarized over the subjects who participated in two or more experiments. Three subjects participated in both Experiment 1 and Experiment 2. The motion distributions for these subjects were similar to those shown in Figure 1, suggesting that the differences obtained were not due to differ-
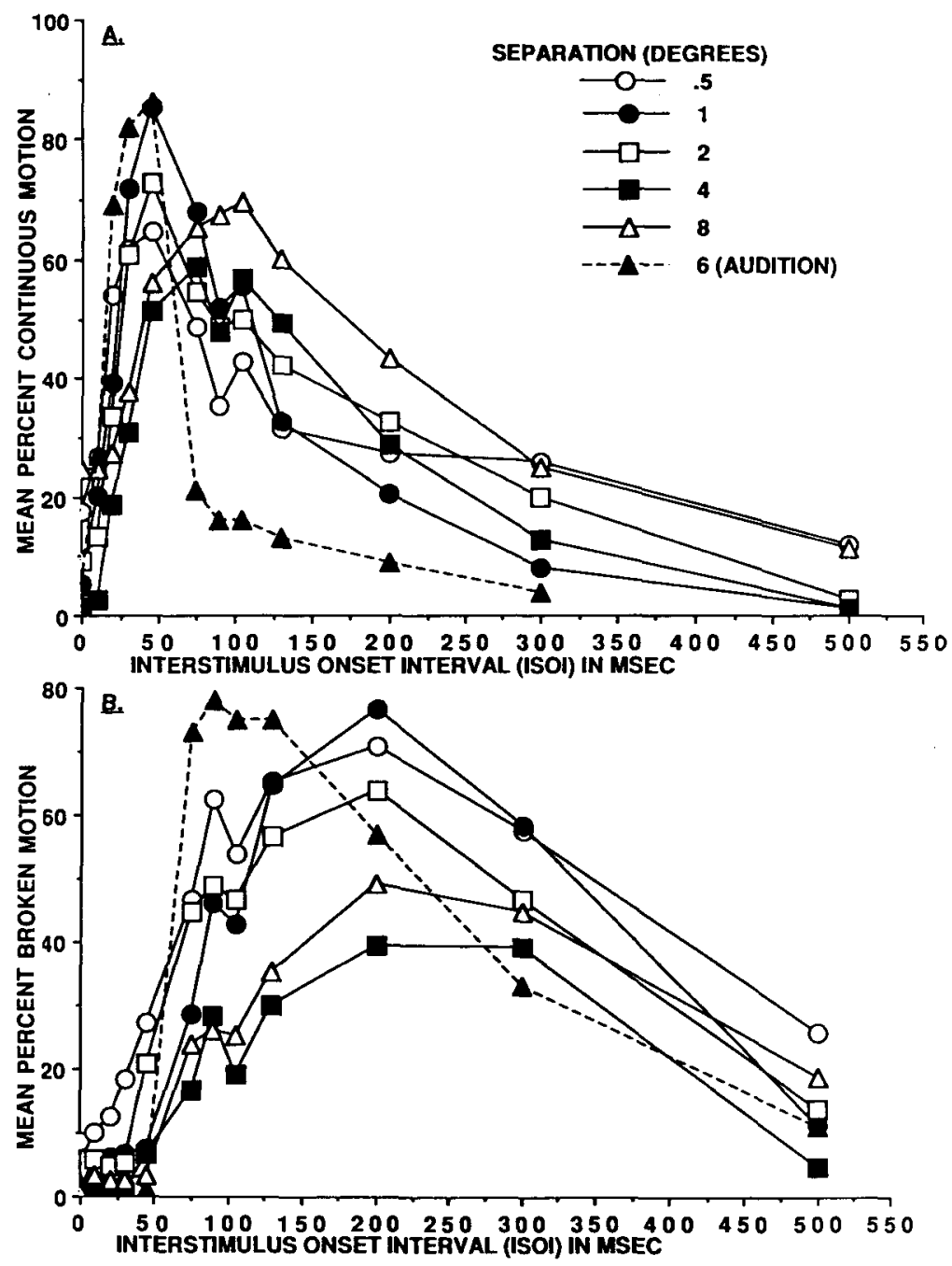

Figure 3. Mean percentage of continuous and broken motion reports for Experiment 3 (visual apparent motion at small spatial separations). The results from Experiment 1 (auditory apparent motion) at $6^{\circ}$ have been included for comparison (A: continuous motion; B: broken motion). 
ences between subjects. The major difference between the experienced and the naive subjects is that more motion was reported by the experienced subjects in the visual modality. ${ }^{3}$ For example, at a separation of $6^{\circ}$, the mean percentage of motion obtained at an ISOI of $45 \mathrm{msec}$ is $84 \%$ for the experienced group, compared to $52 \%$ for the naive group. However, as the separation increased, the percentage of motion decreased for both groups of subjects. Similar observations can be made about the broken motion distributions. Broken motion was seen at the same range of ISOIs, but overall, more motion was reported by the experienced subjects.

Figure 4 presents the average percentages of continuous and broken motion reports for the 2 subjects who participated in all three experiments. In the visual modality, the curves were averaged across separations of $2^{\circ}$ or less and across separations greater than $2^{\circ}$. Auditory distributions are included for separations of $6^{\circ}$ and $160^{\circ}$. As is shown in Figure 4A, two distinct motion functions are suggested. For separations of less than $2^{\circ}$, the distribution is more leptokurtotic than the distributions at larger separations, and it is similar to the auditory distribution. For $2^{\circ}$ or less, motion was reported primarily at 30 and $45 \mathrm{msec}$. At larger values of separation, the curve is flatter; its peak extends from $45-130 \mathrm{msec}$. The auditory curves presented are from Experiment 1, with separations of $6^{\circ}$ and $160^{\circ}$. No difference in these distributions is evident. The broken motion functions, shown in Figure 4B, also demonstrate the effect of separation. For separations of less than $2^{\circ}$, the broken motion distribution
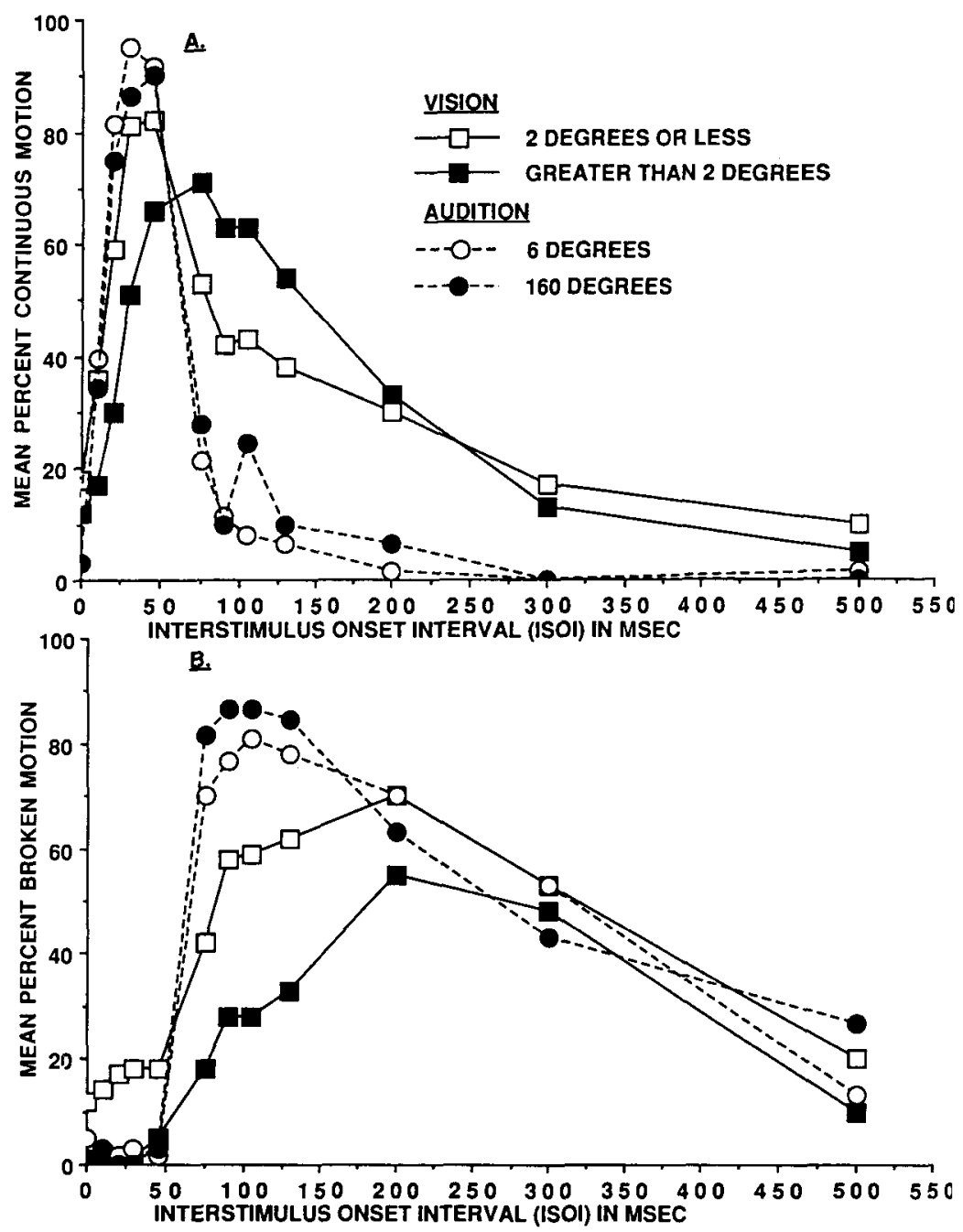

Figure 4. Mean percentage of continuous and broken motion reports for 2 subjects who participated in all three experiments. The auditory distributions presented are for separations of $6^{\circ}$ and $160^{\circ}$. The visual distributions are averaged across separations of $2^{\circ}$ or less and across separations greater than $2^{\circ}$ (A: continuous motion; B: broken motion). 

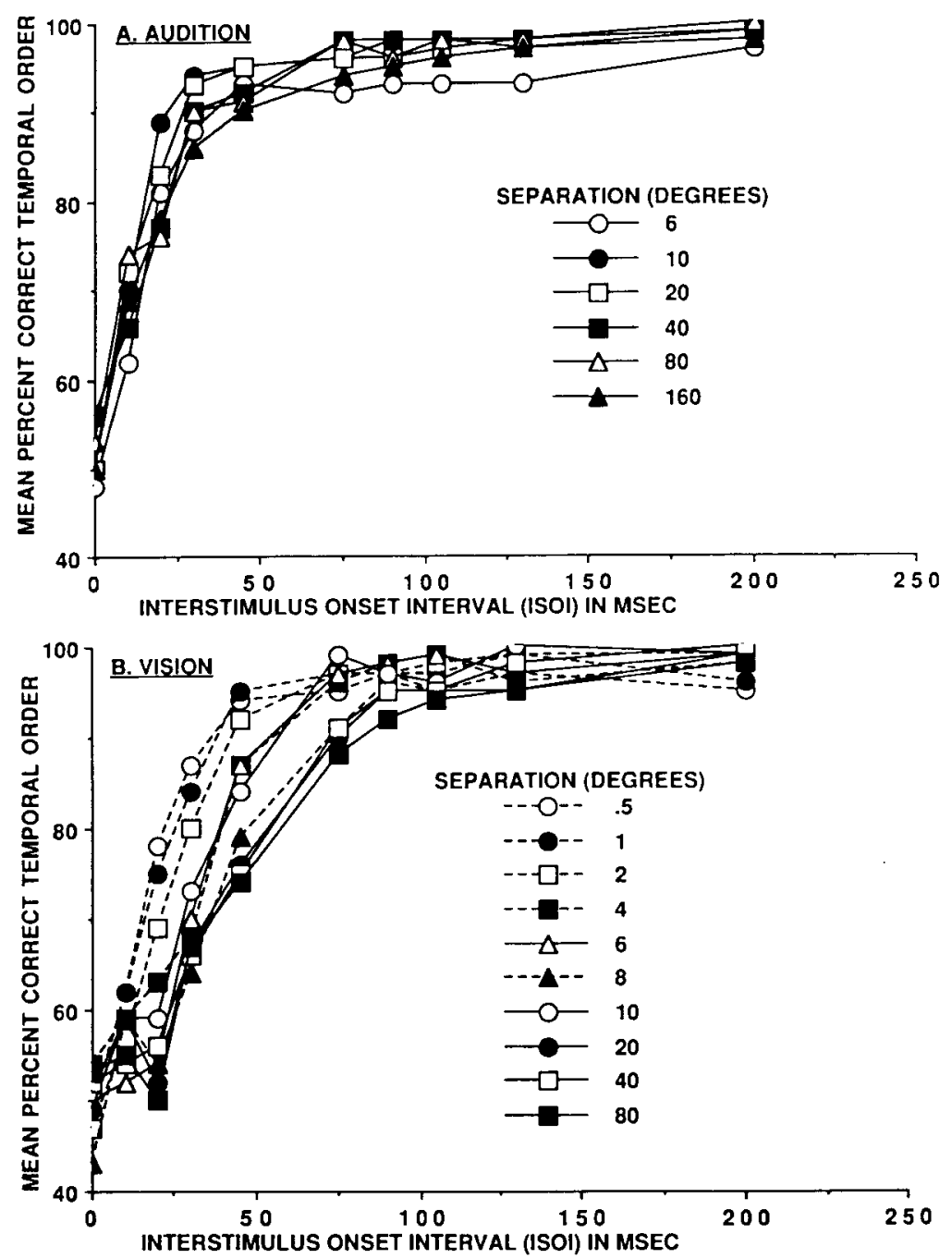

Figure 5. Mean percentage of correct temporal order judgments for Experiments 1-3. A: results of Experiment 1 (auditory condition); B: results of Experiments 2 and 3.

extends across a larger range of ISOIs (130-300 msec). For the larger separations, the peak of the curve is at $200 \mathrm{msec}$, and less broken motion is reported overall.

Figure 5 shows the percentage of correct temporal order judgments, for all three experiments. Figure 5A shows the results from the auditory modality (Experiment 1), and Figure 5B shows the results from the visual modality (Experiments 2 and 3). Overall, subjects were able to determine the correct sequence of stimuli at a lower ISOI in audition. On the average, subjects attained a $75 \%$ correct performance rate at an ISOI of $14 \mathrm{msec}$, and this value was minimally affected by the separation between the sources. In vision, separation did affect this judgment, as is shown in Figure 5B. Three distinct functions are evident. For separations of $2^{\circ}$ or less, $75 \%$ correct performance was achieved at an ISOI of $32 \mathrm{msec}$. For separations of $4^{\circ}-10^{\circ}, 75 \%$ correct performance was reached at an ISOI of $36 \mathrm{msec}$. Finally, for separations greater than $10^{\circ}$, an ISOI of $45 \mathrm{msec}$ produced better than chance performance.

\section{DISCUSSION}

The results from these three experiments on auditory and visual apparent motion can be summarized as follows: First, the overall values of ISOI that produce the perception of auditory continuous or broken motion are smaller than the values that produce the same experience in vision, provided that the conditions are similar. The range of ISOIs under which the perception of continuous and broken motion is produced is also smaller in audition. Second, spatial separation seems to affect the ISOI range more in vision than in audition. In fact, at separations of $2^{\circ}$ or less, the peak of the visual motion function occurs at the same ISOI value as does the peak of the auditory function. Finally, judgments of temporal order are affected by the separation in vision, but separation has no effect in audition.

Auditory apparent motion is produced at smaller values of ISOI than visual apparent motion is, and the overall range of ISOIs that produce the phenomenon is more re- 
stricted in the auditory modality. This observation agrees with previous reports on auditory apparent motion (e.g., Briggs \& Perrott, 1972; Burtt, 1917a). However, our results suggest that conditions can be arranged to produce nearly equivalent experiences at the same ISOI. In Experiment 3 , it was shown that for very small values of separation, the peaks of the visual motion curves are located at the same ISOI as that for the peak of the auditory curve at $6^{\circ}$. It is possible, therefore, that at some very small value of separation, apparent motion would be produced by an identical range of ISOIs. Unfortunately, we could not achieve smaller separations with our apparatus.

When the separations are equal, however, auditory apparent motion occurs at smaller values of ISOI. One might explain these differences in terms of the differences between temporal acuities in the two modalities. Temporal acuity has been measured in many different ways. One can examine the ability of subjects to distinguish between simultaneity and successiveness, for example. In a review of the literature on this topic, Hirsh and Sherrick (1961) report that the visual system is more sluggish than the auditory system is on this task. Two auditory events can be detected as being successive if the interval between them is at least 2 msec, but two visual events must be separated by at least $44 \mathrm{msec}$ if they are to be seen as successive. Therefore, the visual function is 20 times poorer than the auditory function on the temporal acuity task.

In our data, the largest difference between audition and vision in the peaks of the motion functions represents only a twofold increase in the ISOI, so our results cannot be accounted for by differences in temporal acuity. This is not surprising, for the perception of motion must involve both spatial and temporal acuity. Our results indicate further that the influence of spatial and temporal factors depends on the modality of presentation. In is unlikely, therefore, that either spatial or temporal acuity alone could account for the differences between auditory and visual apparent motion that we obtained.

The second major difference between auditory and visual apparent motion that we found involves the effect of the separation between the stimuli. In the auditory modality, separation had virtually no effect on the ISOI range that produced continuous and broken motion. In the visual modality, separation affected the motion functions differently at very small and at very large values.

These effects of separation are shown in Table 1, which presents the optimal range of ISOIs for continuous and broken motion at each separation and modality. This range was determined by finding the ISOI that produced the maximum mean percentage of continuous or broken motion reports, and by finding the range of ISOIs that elicited motion reports within $20 \%$ of the maximum value. In addition, the percentage of reports of each motion category was averaged across this ISOI range. We considered the percentage of motion reports to be a measure of the relative strength of the motion in this range.

In audition, as Table 1 indicates, separation had no effect on the ISOI range that produced motion. Motion was seen for ISOIs between 20 and $45 \mathrm{msec}$ for every sepa-
Table 1

Optimal ISOI Range for Auditory and Visual Continuous and Broken Apparent Motion at all Separations Tested

\begin{tabular}{|c|c|c|c|c|}
\hline \multirow[b]{2}{*}{ Separation } & \multicolumn{2}{|c|}{ Continuous Motion } & \multicolumn{2}{|c|}{ Broken Motion } \\
\hline & $\begin{array}{l}\text { ISOI } \\
\text { Range }\end{array}$ & $\begin{array}{l}\text { Mean \% } \\
\text { Reported }\end{array}$ & $\begin{array}{c}\text { ISOI } \\
\text { Range }\end{array}$ & $\begin{array}{l}\text { Mean \% } \\
\text { Reported }\end{array}$ \\
\hline \multicolumn{5}{|c|}{ Audition } \\
\hline $6^{\circ}$ & $20-45$ & 78 & $75-130$ & 74 \\
\hline $10^{\circ}$ & $20-45$ & 88 & $75-130$ & 79 \\
\hline $20^{\circ}$ & $20-45$ & 78 & $75-200$ & 60 \\
\hline $40^{\circ}$ & $30-45$ & 83 & $75-300$ & 57 \\
\hline $80^{\circ}$ & $20-45$ & 72 & $75-200$ & 61 \\
\hline $160^{\circ}$ & $30-45$ & 73 & $75-200$ & 44 \\
\hline \multicolumn{5}{|c|}{ Vision } \\
\hline $.5^{\circ}$ & $20-75$ & 56 & $90-300$ & 61 \\
\hline $1^{\circ}$ & $30-75$ & 74 & $130-300$ & 66 \\
\hline $2^{\circ}$ & $30-75$ & 62 & $75-300$ & 50 \\
\hline $4^{\circ}$ & $45-130$ & 52 & $90-300$ & 31 \\
\hline $6^{\circ}$ & $45-130$ & 57 & $90-300$ & 52 \\
\hline $8^{\circ}$ & $45-130$ & 63 & $130-300$ & 42 \\
\hline $10^{\circ}$ & $45-130$ & 60 & $130-300$ & 52 \\
\hline $20^{\circ}$ & $45-130$ & 54 & $105-300$ & 52 \\
\hline $40^{\circ}$ & $45-130$ & 43 & $90-300$ & 52 \\
\hline $80^{\circ}$ & $30-130$ & 42 & $105-300$ & 33 \\
\hline
\end{tabular}

Note-ISOIs (interstimulus onset intervals) are given in milliseconds.

ration tested. In addition, the overall percentage of motion reports decreased only slightly. Broken motion was heard at ISOIs between 75 and $130 \mathrm{msec}$ for separations less than $10^{\circ}$, and the upper limit was extended out to 200-300 msec at larger separations.

Since the maximum obtainable separation is $180^{\circ}$, it would appear that auditory apparent motion can be heard at all possible values of separation. However, as indicated in the overall percentage of motion reported, there is a slight reduction in the strength of motion reported at larger separations. This is particularly true for broken motion, where this value decreased by $30 \%$ at $160^{\circ}$ separation.

The separation had a varied effect on the visual illusion. For separations of $2^{\circ}$ or less, the values of ISOIs that produced continuous motion were lower than the values found at larger separations. The optimum range of ISOIs for motion was roughly $30-75 \mathrm{msec}$ at separations of less than $2^{\circ}$. At greater separations, the range shifted to $45-130 \mathrm{msec}$. At separations of $2^{\circ}$ or less, the stimuli were, of course, stimulating only the foveal area of the retina. Therefore, our data suggest that the ISOI range that produces motion is dependent on the area of the retina that is stimulated.

The broken motion functions were not affected by separation. As is indicated in Table 1, there is some variability in the lower ISOI bound for broken motion, although there is no systematic change with separation. In addition, the upper bound is constant at $300 \mathrm{msec}$ at all separations, although very few large ISOIs were employed in our experiments. Under the conditions noted here, broken motion was seen from roughly $90-300 \mathrm{msec}$ at all separations.

When the separation becomes relatively large, however, the strength of the motion seen becomes degraded, as is indicated by the percentages of continuous and broken mo- 
tion reports in the optimum range. In Table 1, at large separations, the overall percentage of continuous and broken motion reports decreases. For both types of motion, this overall percentage dropped by roughly $30 \%$ from the best separation $\left(1^{\circ}\right)$ to the $80^{\circ}$ condition. This suggests a limit on the maximum value of separation at which visual apparent motion can be seen. Furthermore, as this limit is approached, the perception of continuous motion seems to change to a perception of broken motion before the motion is lost completely.

The results from the visual experiments (Experiments 2 and 3) appear to support Korte's (1915) assertion that as the separation increases, the ISOI must also increase if the experience of motion is to be maintained. However, our results suggest that the effect of separation is related to the area stimulated on the retina. Within the particular visual system stimulated, separation had little if any effect on the ISOI range. The results of Experiment 1 supported the finding of Strybel et al. (1989) that for binaural listening conditions, there is no effect of separation on the ISOIs that produce the auditory apparent motion. Therefore, under nearly identical experimental conditions, the spatial separation between the stimuli affected the ISOI range differently in each modality.

At this time, many possible explanations could be offered for the different effects of separation on apparent motion in vision and audition, simply because very little is known about the processing of auditory motion in general. One possible interpretation will be proposed here. If one assumes that the effect of separation depends on the distance between the receptors that are stimulated, then in our auditory experiment we failed to manipulate separation. In order to affect separate receptor sites on the basilar membrane, one would have to vary the frequencies contained in each stimulus.

Preliminary data (Smith \& Strybel, 1988) have suggested this observation. Using pure tones of different frequencies to produce dichotic auditory apparent motion, we attempted to determine the maximum frequency difference between two pure tones that would allow that apparent motion to be heard. The maximum frequency difference that resulted in apparent motion was found to be a function of the ISOI used. At an ISOI of $20 \mathrm{msec}$, the maximum frequency difference that produced the experience of motion was less than the maximum difference at $50 \mathrm{msec}$. These results are based on a narrow range of frequencies and ISOIs, however. We are currently attempting to extend this finding over a larger range. The results suggest an interaction between frequency and ISOI that may be analogous to the interaction between separation and ISOI in the visual modality.

The third major difference between auditory and visual motion that we found in the present experiments is related to the judgment of temporal order. In the auditory modality, our subjects were able to determine the correct order of presentation when the ISOI exceeded $14 \mathrm{msec}$, and this value appears to have been unaffected by the separation of the stimuli. On the other hand, performance on visual temporal order tasks was much poorer and was af- fected by the separation between stimuli. Three values were obtained, depending on the separation. For separations of less than $2^{\circ}$, a mean ISOI of $32 \mathrm{msec}$ was required for correct temporal order determination. For separations of between $4^{\circ}$ and $10^{\circ}, 36 \mathrm{msec}$, and for larger separations, $45 \mathrm{msec}$ were required for temporal order determination.

The finding of a difference in the ISOI value that allows proper temporal order discrimination may at first appear to contradict the vast literature on temporal order judgments (e.g., Hirsh \& Sherrick, 1961), which indicates that the proper temporal order of two stimuli can be determined if they are separated by an ISOI of roughly $20 \mathrm{msec}$, regardless of modality. However, our results violated one of the conditions for a true temporal order task. According to Hirsh and Sherrick, the constant value of $20 \mathrm{msec}$ is obtained when no spatial interactions are present. The authors mention that when motion is produced by the stimulus arrangement, the correct temporal order can be inferred at lower ISOIs simply by determining the direction of motion. This observation has been previously verified for the auditory modality (Perrott, Strybel, \& Manligas, 1987). It is not surprising, therefore, that visual temporal order judgments were affected by spatial separation, since the percentage of motion reports was also affected by this variable.

In summary, we have examined auditory and visual apparent motion under nearly identical experimental conditions. Auditory apparent motion is generally produced by smaller ISOIs than visual apparent motion is, and it is not affected by the spatial separation between stimuli. The ISOIs that produce visual apparent motion depend on the spatial separation between stimuli. At very small separations, the ISOI values that produce visual motion overlap with those that produce auditory motion. Thus, under certain conditions, motion in vision and in audition may be produced by the same values of the ISOI.

\section{REFERENCES}

ANstis, S. M. (1978). Apparent movement. In R. Held, W. Leibowitz, \& H. Teuber (Eds.), Handbook of sensory physiology: Vol. 8. Perception. Berlin: Springer-Verlag.

BAKER, C. L., \& BRADDICK, O. J. (1985). Temporal properties of the short-range process in apparent motion. Perception, 14, 181-192.

Braddick, O. J. (1974). A short-range process in apparent motion. Vision Research, 14, 519-528.

Briggs, R., \& Perrot, D. R. (1972). Auditory apparent movement under dichotic listening conditions. Jourmal of Experimental Psychology, 92, 83-91.

BURTT, H. E. (1917a). Auditory illusions of movement-A preliminary study. Journal of Experimental Psychology, 2, 63-75.

BURTT, H. E. (1917b). Tactual illusions of movement. Journal of Experimental Psychology, 2, 371-385.

EXNER, S. (1875). Über das Sehen von Bewegung. Wiener. Sitz.-Ber. 72, ABT 3.

Hirsh, I. J., \& Sherrick, C. E. (1961). Perceived order in different sense modalities. Journal of Experimental Psychology, 62, 423-432.

Kolers, P. A. (1972). Aspects of motion perception. Oxford, England: Pergamon Press

KORTE, A. (1915). Kinematoskopische Untersuchungen. Zeitschrifi fur Psychologie, 72, 193-206.

Neuhaus, W. (1930). Experimentelle Untersuchungen der Schein- 
bewegung. Pflügers Archiv für die Gesamte Psychologie des Menschen und der Tiere, 75, 415-458.

Perrotr, D. R. (1974). Auditory apparent motion. Journal of Auditory Research, 14, 163-169.

Perrott, D. R., Strybel, T. Z., \& Manligas, C. L. (1987). Conditions under which the Haas precedence effect may or may not occur. Journal of Auditory Research, 27, 59-72.

SmITH, C. B., \& STRYBEL, T. Z. (1988, April). Frequency effects in auditory apparent motion. Paper presented at the meeting of the Western Psychological Association, Burlingame, CA.

Strybel, T. Z., Manligas, C. L., \& Perrott, D. R. (1989). Auditory apparent motion under binaural and monaural listening conditions. Perception \& Psychophysics, 45, 371-377.

Wertheimer, M. (1912). Experimentelle Studien über das Sehen von Bewegung. In T. Shipley (Ed. and Trans.), Classics in psychology (pp. 1032-1089). New York: Philosophical Library.

\section{NOTES}

1. Temporal separation can be described in terms of the interstimulus interval (ISI) or the interstimulus onset interval (ISOI). Which term is appropriate is a matter of debate (see, e.g., Kolers, 1972). In the present investigation, both measures would describe the findings adequately; ISOI was chosen for convenience.

2. To achieve even greater similarity in experimental conditions, one could attempt to match the brightness of the light with the loudness of the sound. Since the intensity of the stimulus does not affect visual apparent motion (Neuhaus, 1930), however, the levels used in the present investigation were selected only to provide a readily detectable stimulus in each modality.

3. Kolers (1972) noted two effects of practice on the perception of visual apparent motion: Practice can reduce the range of ISOIs and extend the range of conditions under which apparent motion is perceived. In the present experiment, the second effect was noted; all of the experienced subjects perceived more motion at all separations. However, this effect of practice was obtained between modalities.

(Manuscript received April 25, 1988; revision accepted for publication November $21,1989$. 